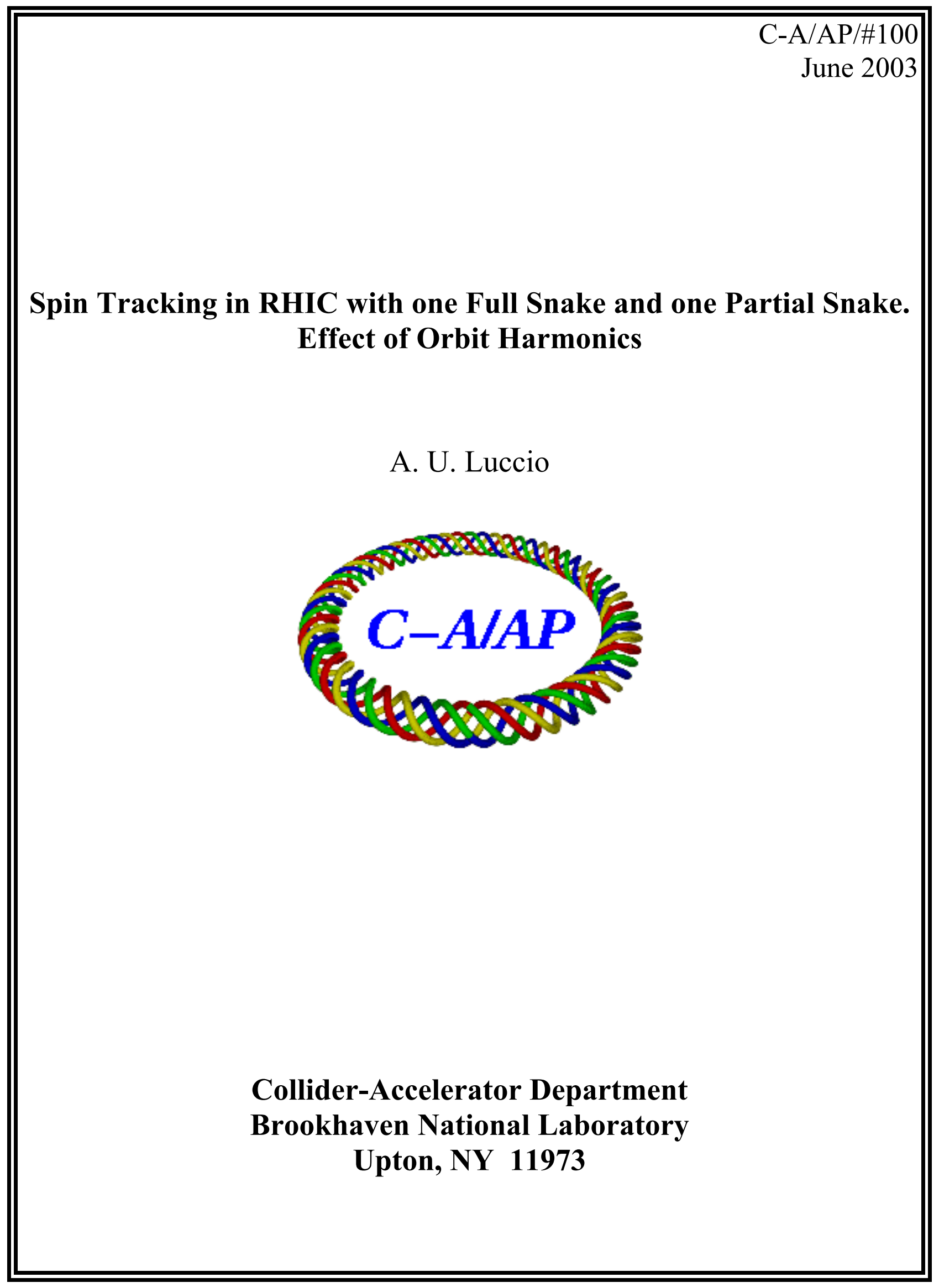




\title{
Spin Tracking in RHIC with one Full Snake and one Partial Snake. Effect of Orbit Harmonics
}

\author{
A.U. Luccio \\ June 25, 2003
}

\section{Introduction}

Both rings in RHIC, Blue and Yellow are furnished with two helical full snakes to allow the acceleration of polarized protons to the highest energy of the machine with minimal polarization loss. During the polarized proton run in the Spring of 2003 the coils of the central sections of one of the two snakes of the Yellow Ring were damaged, so the snake could not be operated as a full snake. However, by adjusting the currents in the outer snake coils, this snake could still be used as a partial snake. The polarization of the beam could still be preserved, altough to a lesser degree. This report describes tracking of the spin with the code Spink in RHIC-Yellow, with one full snake and one partial snake, to show what are the value of the beam polarization that can be obtained in these conditions.

\section{Spin rotation in the snakes}

The snakes in RHIC are made up of four helical dipoles. If all helices are working properly, the snake is rotating the spin by $\mu=180^{\circ}$, from the vertical up to down (or vice-versa) around an axis laying in the horizontal plane and making an angle of $\pm 45^{0}$ with the longitudinal azis $z$. The angle $\mu$ is independent of the energy of the protons. This situation is shown in Fig. 1. Field, orbit and spin components in a partial snake, with no inner helices, are shown in Fig. 2. This snake is set to produce a spin rotation of $\mu=158^{0}$ around an axis at $-45^{0}$. The orbit has a vertical excursion that depends on energy: maximum at the lowest energy in RHIC (the figure shows a vertical bump of about $5 \mathrm{~cm}$ at $\gamma=26$-injection energy). To respect the clearance of the snake, a vertical bump should be put in RHIC-Yellow to upset half of this excursion. The created bump should be of $\sim 4 \mathrm{~cm}$ at injection and should decay completely for $\gamma=50$.

\section{MAD work}

Spink reads the accelerator configuration from MAD. In the present case we wanted to configure MAD to take into consideration the machine imperfections -foremost the measured vertical displacement of the machine magnets- and to correct the orbit in MAD with the Micado algorithm to produce an orbit as horizontal possible. At variance with more common conditions, where the orbit is corrected to make it centered in the machine multipoles, this is the best condition to preserve polarization: spin orientation is best preserved when carried by particles moving in an horizontal plane. In MAD, the commands to achieve this condions are listed in Table 1.

After the output of MAD is obtained, we run the Spink pre-processor mad-read to prepare the input to Spink in the form of a file -call it "xxx.sy" - containing the transfer maps from MAD, the Twiss functions, the errors, the COD orbit and finally the one-turn orbit map, made symplectic. An example of entry in that file is given in Table A. 


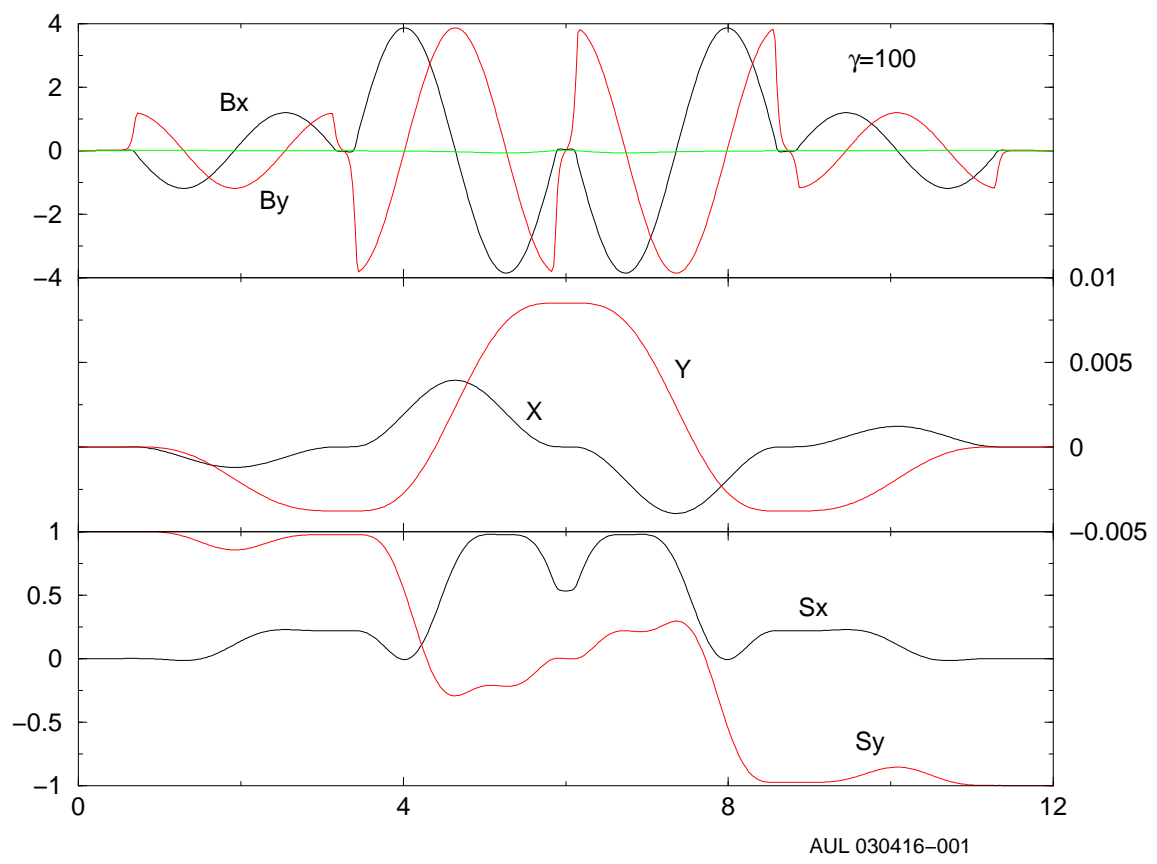

Figure 1: Full snake. From top to bottom: Field, orbit, spin. Proton energy $\gamma=100$. Spin and field along the trajectory.

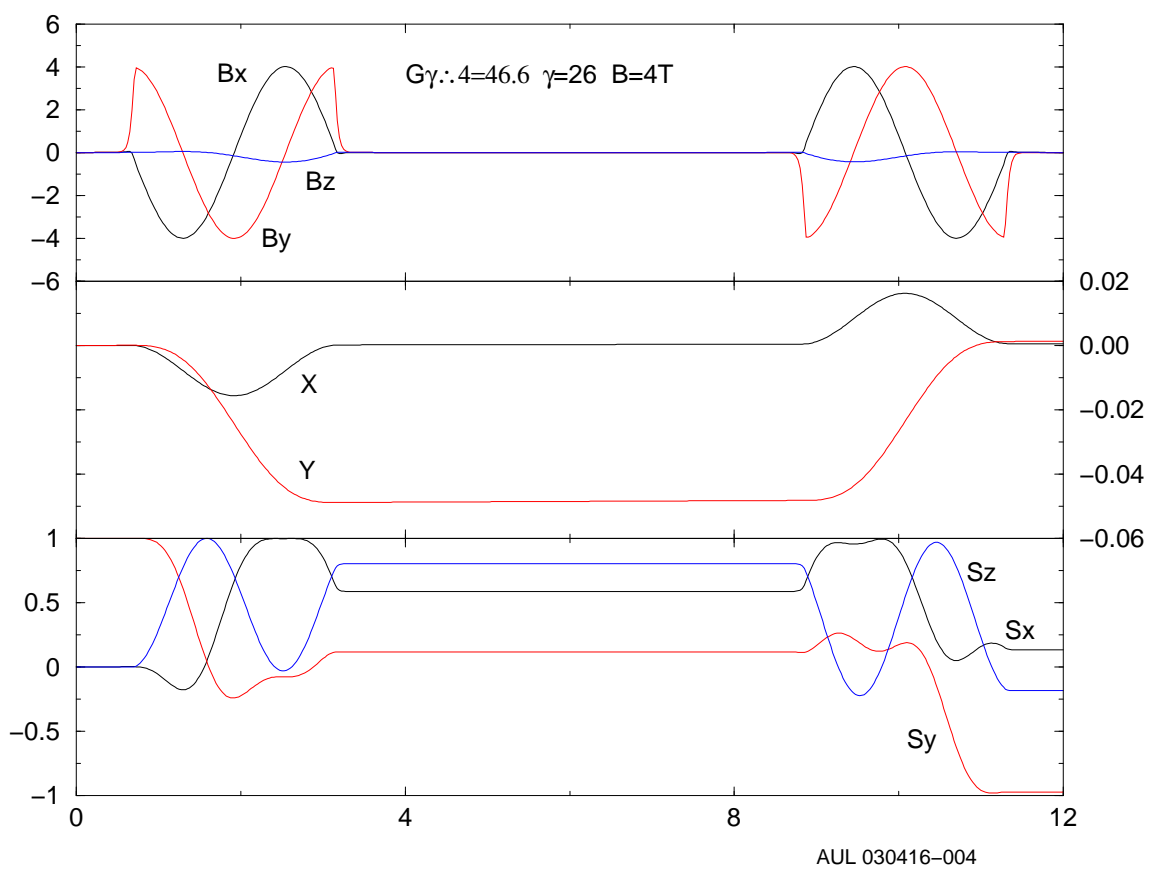

Figure 2: partial snake. Field, orbit, spin. Proton energy $\gamma=26$ 


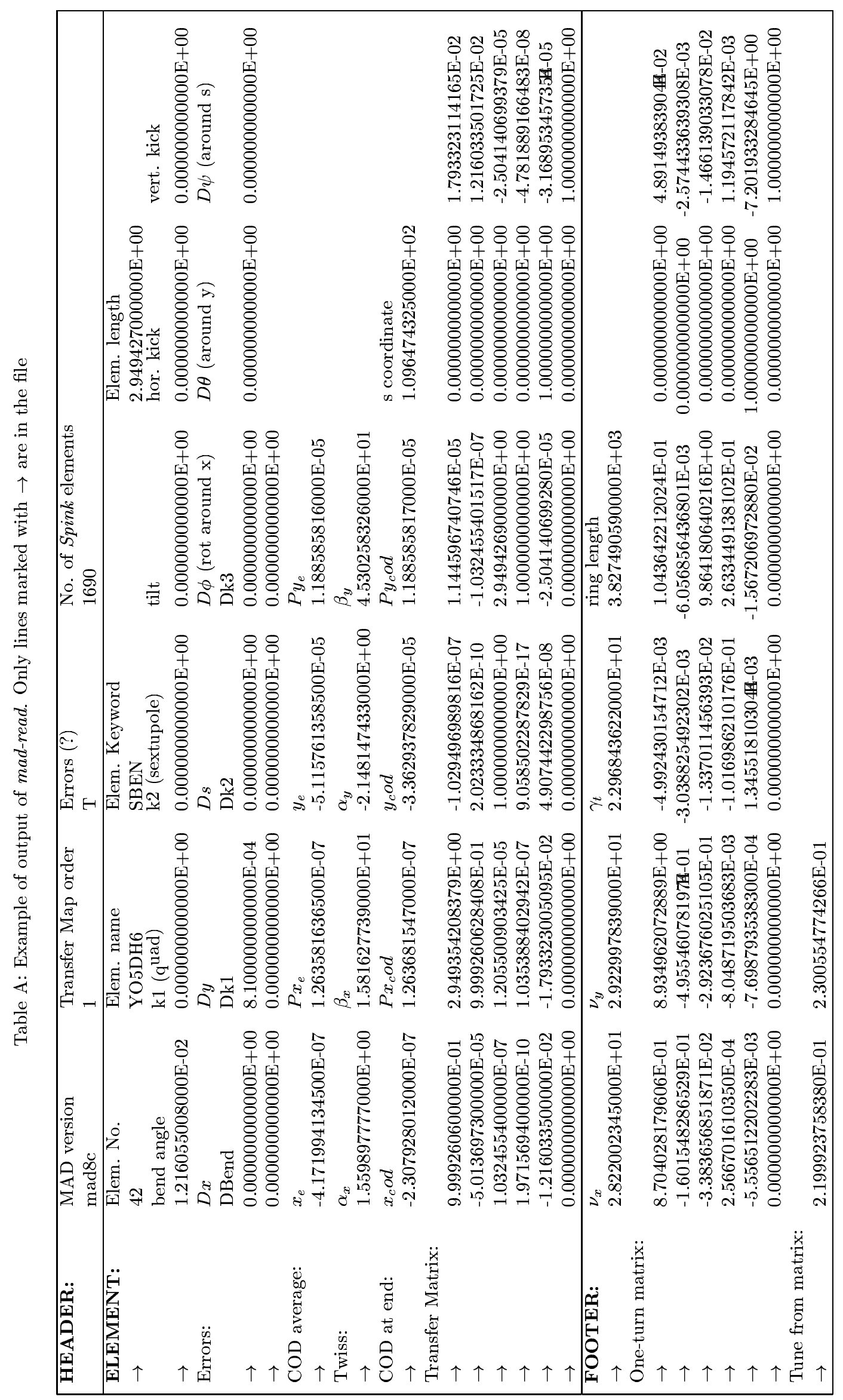


Table 1: MAD8 commands as in file xxx.mad, see diagram of Fig. 3

\begin{tabular}{|ll|}
\hline command & result \\
\hline YMON $:=0$ & set reference level for BPMs \\
USE, RHIC & ("RHIC" set to "Yellow") \\
CALL, filename = 'align-mad.dat' & file containing alignment errors \\
SETOPTS, echo & set up echo file \\
CORRECT, error $=3 . e-4$, iterate $=10$ & invoke Micado \\
c2list, m1list ,m2list & print list of correctors and monitors \\
SELECT, flag=FIRST, range =full & write first order transfer maps to echo file \\
PRINT, RHIC & set up output file .madout \\
TWISS, tape='yellow.twiss' & calculate twiss values and print optics to a file \\
SELECT, error ,range=full & use errors \\
EPRINT,range=full & print imperfection table to madout \\
\hline
\end{tabular}

The complete cycle of codes/pre-processors for this process is diagrammatically shown in Fig. 3.

\section{Spin Tracking in Yellow with Alignment Errors}

Fig. 4 shows the measured vertical alignment errors in Yellow and their FFT. A 1.st and 6.th harmonic are apparent. The curve is best fit with the following function

$$
y=a_{0}+a_{1} \sin \left(6 \frac{2 \pi}{L} s+a_{2}\right)+a_{3} \sin \left(\frac{2 \pi}{L} s+a_{4}\right),
$$

with $L=3827.49$ the length of the machine, and

$$
a_{0}=-0.8604, \quad a_{1}=-0.7728, \quad a_{2}=1.4339, \quad a_{3}=-1.2411, \quad a_{4}=5.6503
$$

The figure also shows a typical measured closed orbit. This orbit is measured at the beginning of the ramp, however it did not change appreciably later in the ramp. The orbit shows some very marked peaks in correspondence to the Intersection Regions, where bumps where applied. Artificially cleaning the IR bumps, the orbits appears as in Fig. 5, together with its harmonic analysis. The figure shows a sizable content of harmonics 1, 2, 6, 12 and 18.

Spink tracks polarized protons through RHIC. We are showing the results of one particle tracking during the acceleration ramp, from injection at $\gamma=26$ to $\gamma=107$ with the machine set for $\beta *=10 \mathrm{~m}$ in all IR's. The particle was selected on the contour of phase space ellipse with emittances 20 and $15 \mathrm{~mm}$-mrad in $x$ and $y$, respectively. Vertical displacement errors in the elements of the machine are included, as well as an orbit bump that offsets the vertical orbit in the partial snake, according to the specification described in Sec. 2. The bump is maximum at injection and decays linearly to zero in 50,000 turns, i.e. when the energy increases from injection to $\gamma=34.9(G \gamma=62.637)$. Fig. 6 shows the behaviour of the vertical component of the spin when using two full snakes, or a full snake and a partial snake. The orbit is corrected with Micado to $y=0$. For both tracking the spin was put in the matched position at the beginning, i.e. the orientation coincident with the stable spin direction at that point in the lattice, found by stroboscopic averaging in Spink. The stable spin axis for the second case has coordinates $\left(S_{x}, S_{y}, S_{z}\right)=(0.106427,0.989719,-0.095544)$.

\section{Orbit Harmonics}

Some of the polarization losses observed during the experiment may have been caused by imperfection resonances that can be simulated by introducing harmonics in the distorted 


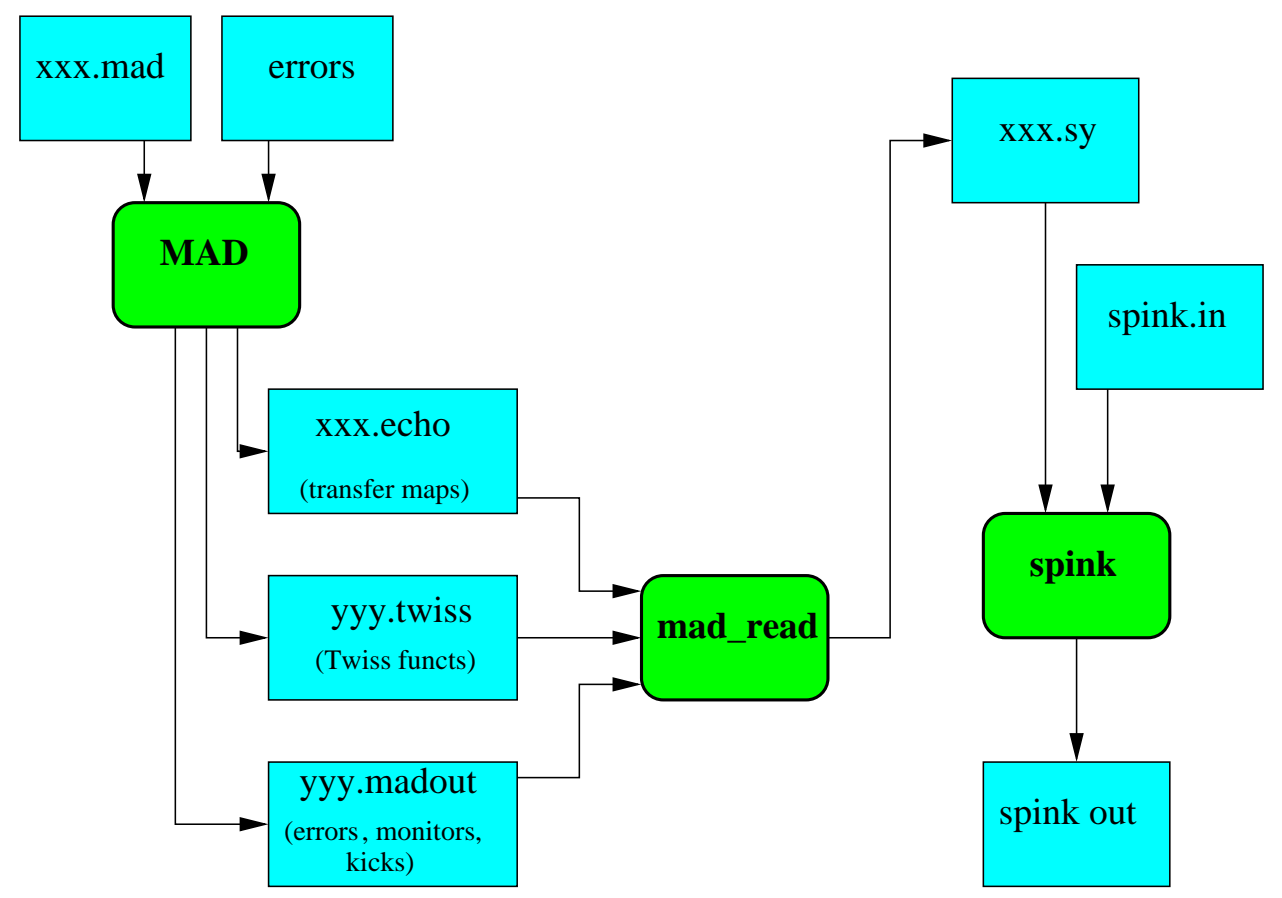

Figure 3: flowchart of the processing of data from $M A D$ to Spink
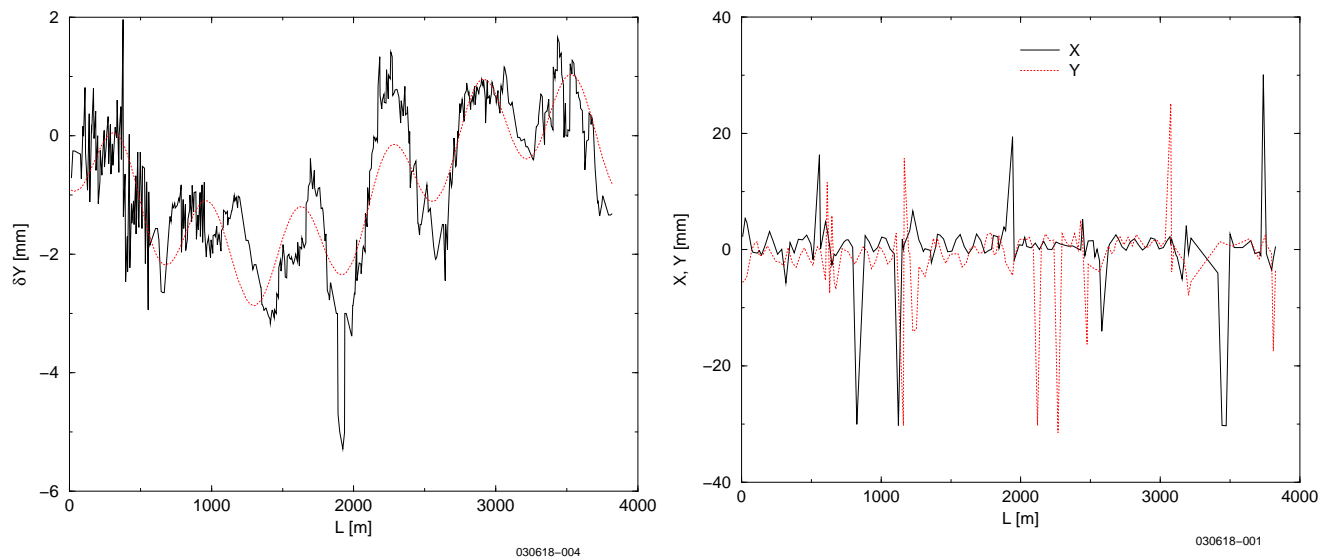

Figure 4: Left: measured vertical misalignment in Yellow and its fitting curve. Right: a measured orbit in Yellow at the beginning of a ramp. IR bumps are shown. 

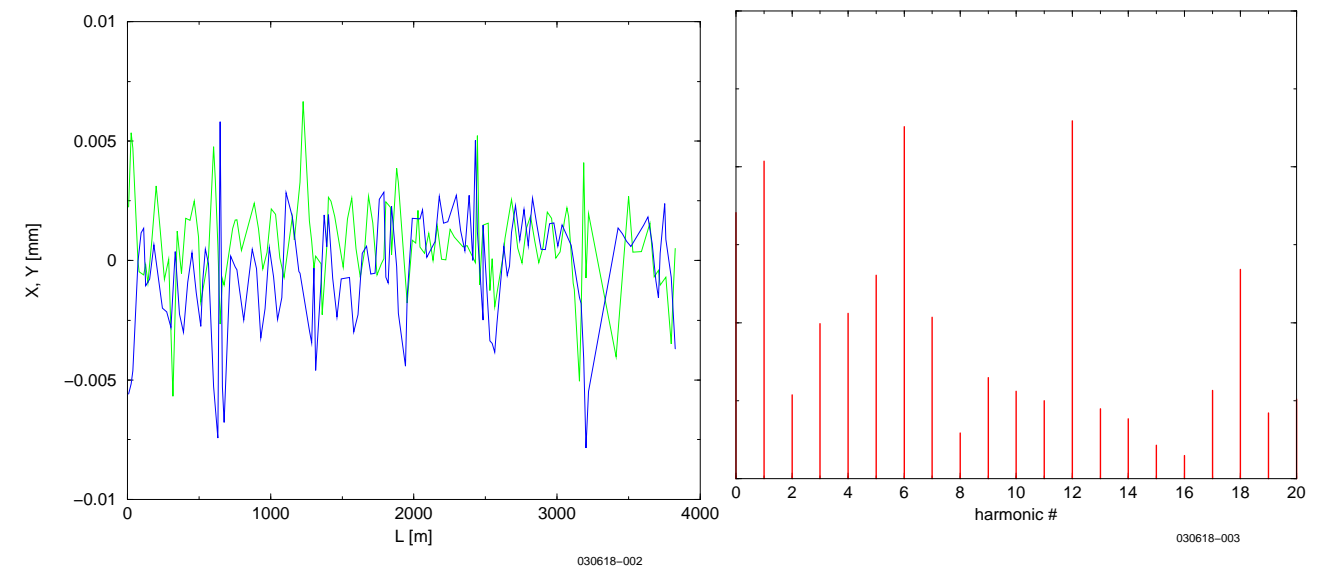

Figure 5: Left: measured orbit in Yellow at the beginning of a ramp. IR bumps removed. Right: FFT of the vertical orbit. Harmonics 1, 2, 6, 12 and 18 are evident.
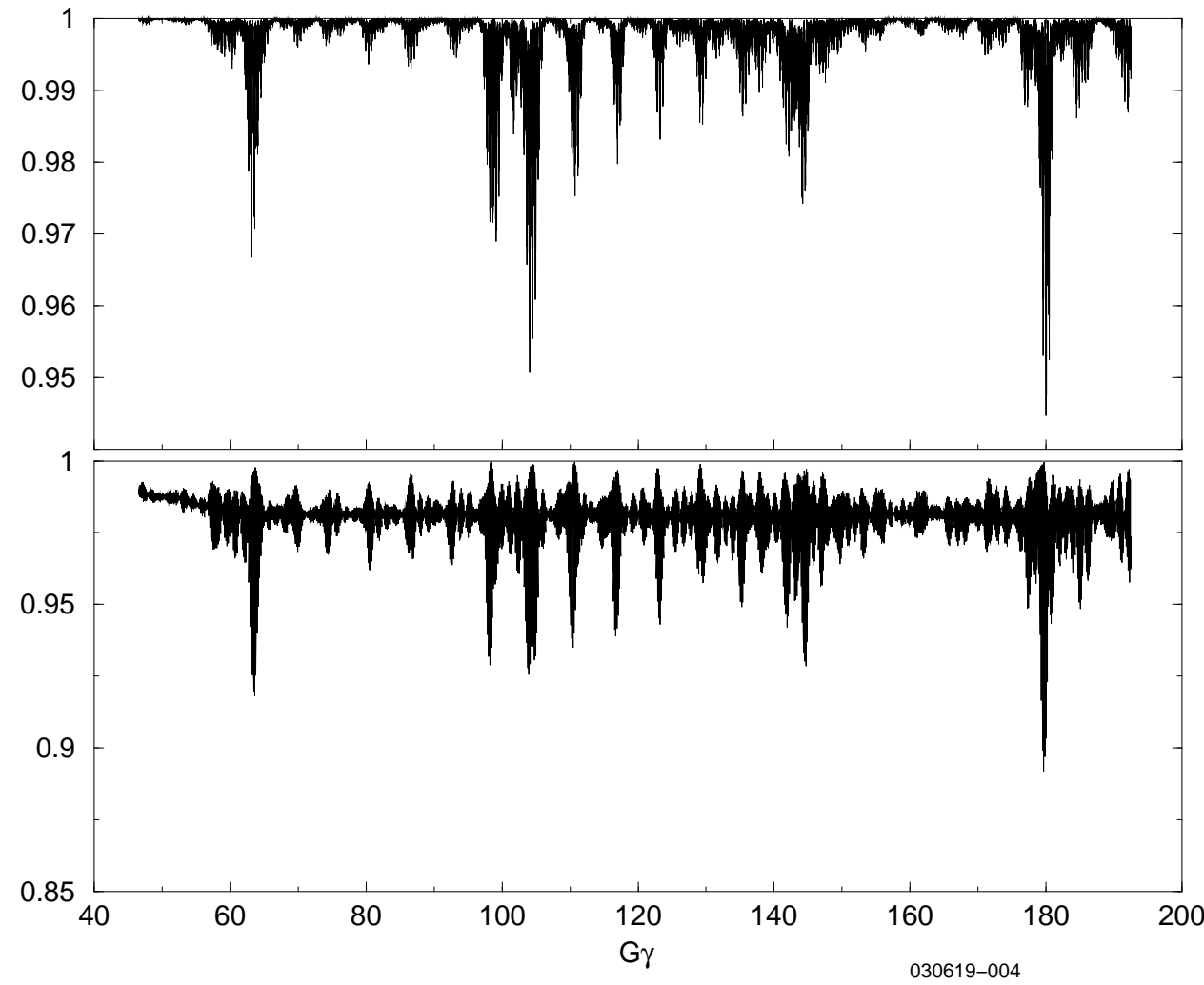

Figure 6: Top: tracking with errors corrected by Micado to $y=0$ plus orbit bump. Use 2 full snakes. Lower: Use a full snake + a partial snake + plus orbit bump.. 

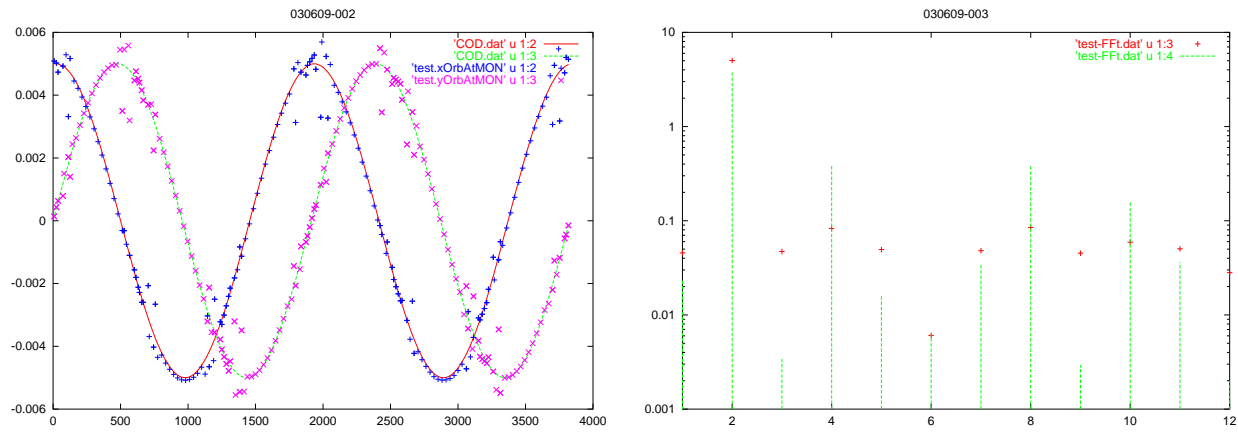

Figure 7: COD with 2.nd harmonic of $5 \mathrm{~mm}$ amplitude in Yellow. No alignment errors. Left: desired COD at BPM's and Micado fit; right: FFT of the curves on the left.
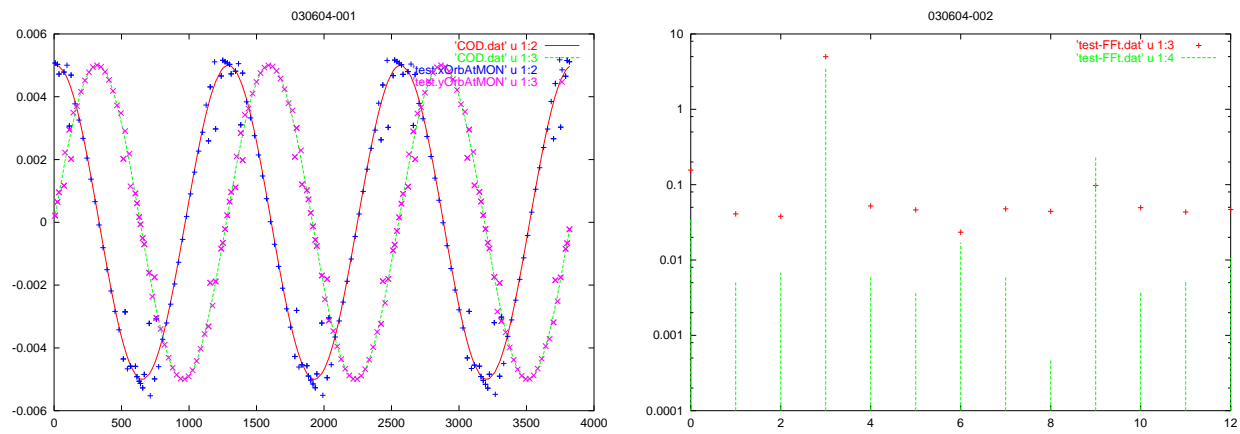

Figure 8: COD with 3.rd harmonic. The rest as in Fig. 7

closed orbit.

We made a series of Spink runs using an error free lattice (no misalignments) and an artificial closed orbit containing some harmonics. To obtain this COD, we put the center of all BPM's in Yellow along the desired closed orbit, using the MAD command EALIGN, and asked MAD to correct the orbit with the MICADO algorithm to pass through the center of the BPM's. Two examples of the results are shown in Figs. 7 and 8

Spink tracking results are shown in Figs. 9 and 10. They show tracking using two full snakes in Yellow, with COD containing various harmonics both in $x$ and $y$ of amplitude 5 and $2 \mathrm{~mm}$, respectively. These figures show that, even with two full snakes, an amplitude of $5 \mathrm{~mm}$ is too large to conserve polarization on the ramp. They also show that even a 2 $\mathrm{mm}$, a 1.st harmonic has an adverse effect the polarization.

Figs. 11 and 12 show tracking for analogous cases, using one full and one partial snake as described before. 


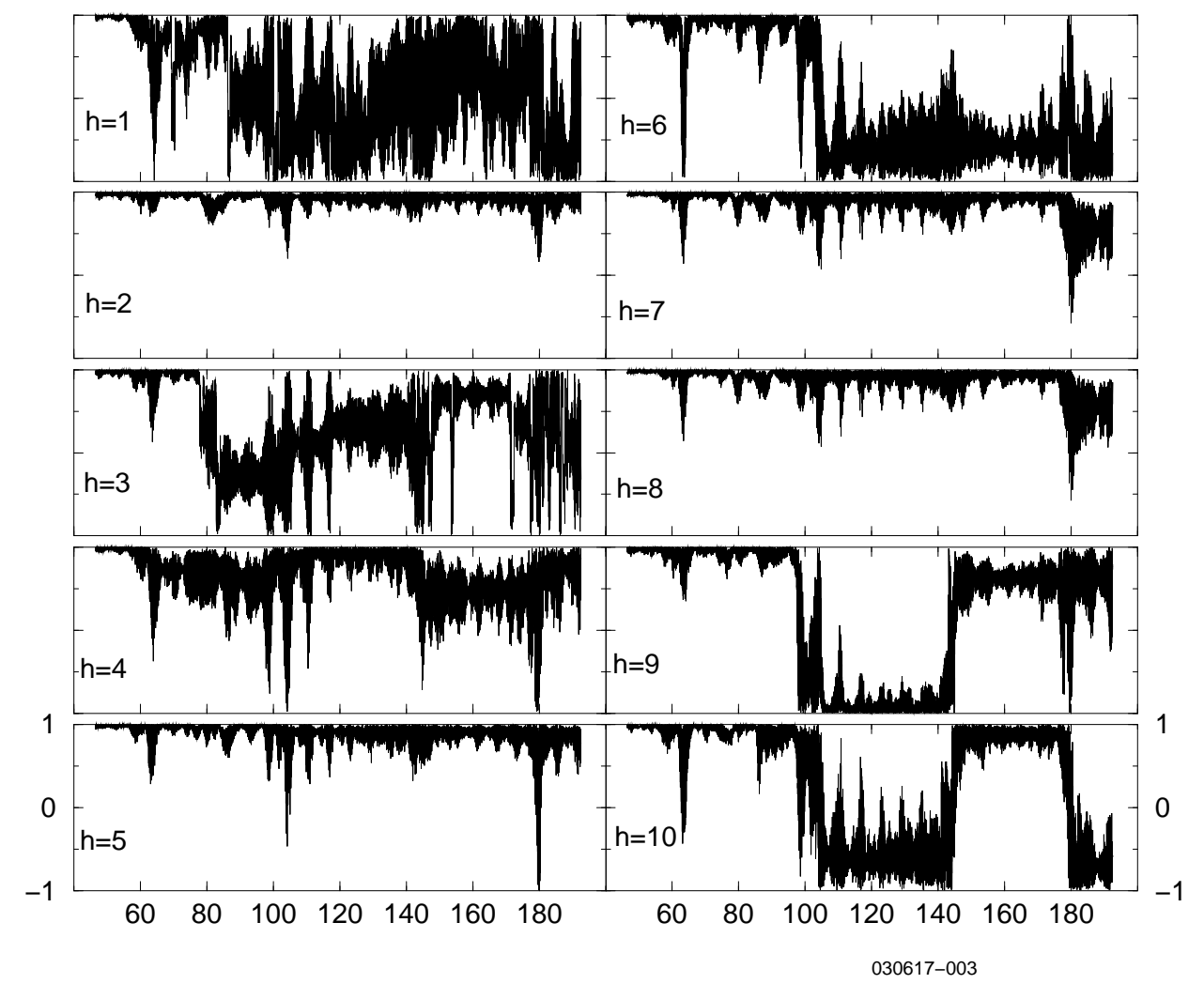

Figure 9: Track in a COD with several harmonic content of $5 \mathrm{~mm}$ amplitude in Yellow. Two full snakes.No alignment errors. Vertical component of spin vs. G|gamma. The vertical scale is the same in all plots. The curves show polarization loss and spin flips in various energy regions. 


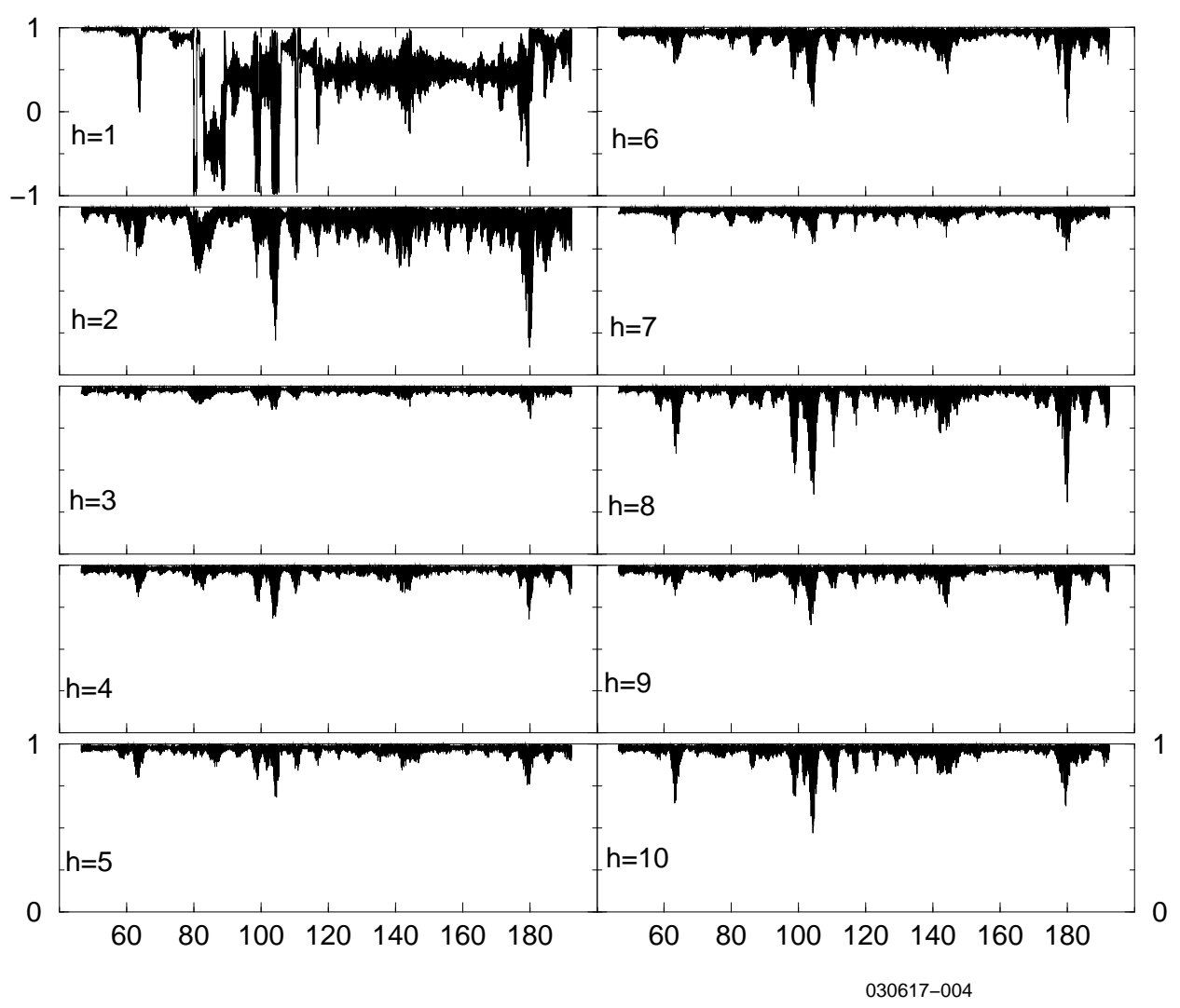

Figure 10: Same as in Fig. 9 with full snakes. $2 \mathrm{~mm}$ COD amplitude. The vertical scale is the same in all plots, but the first for $h=1$. 


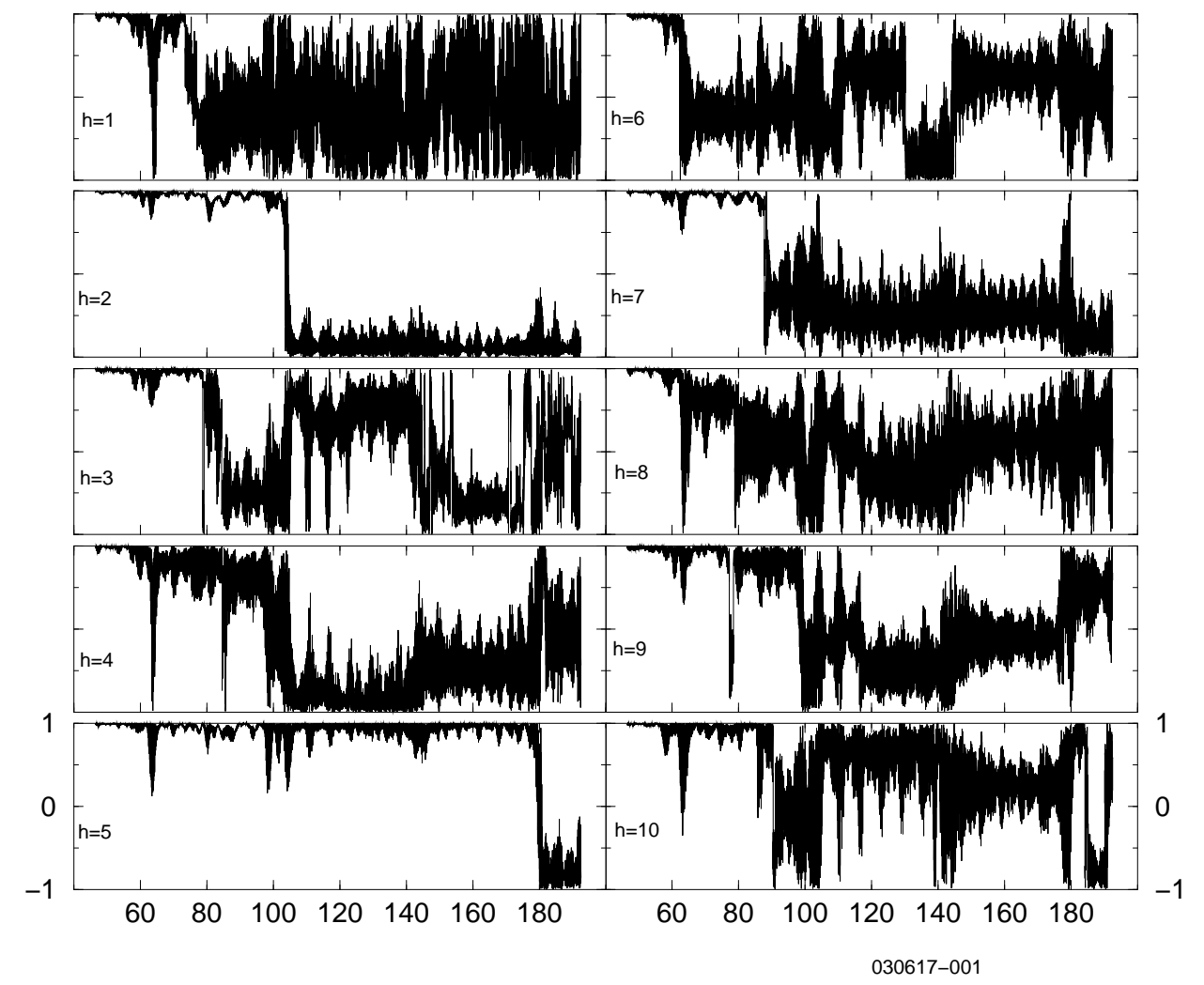

Figure 11: Same as in Fig. 9. $5 \mathrm{~mm}$ COD. One full snake and one partial snake. Same vertical scale in all plots. 


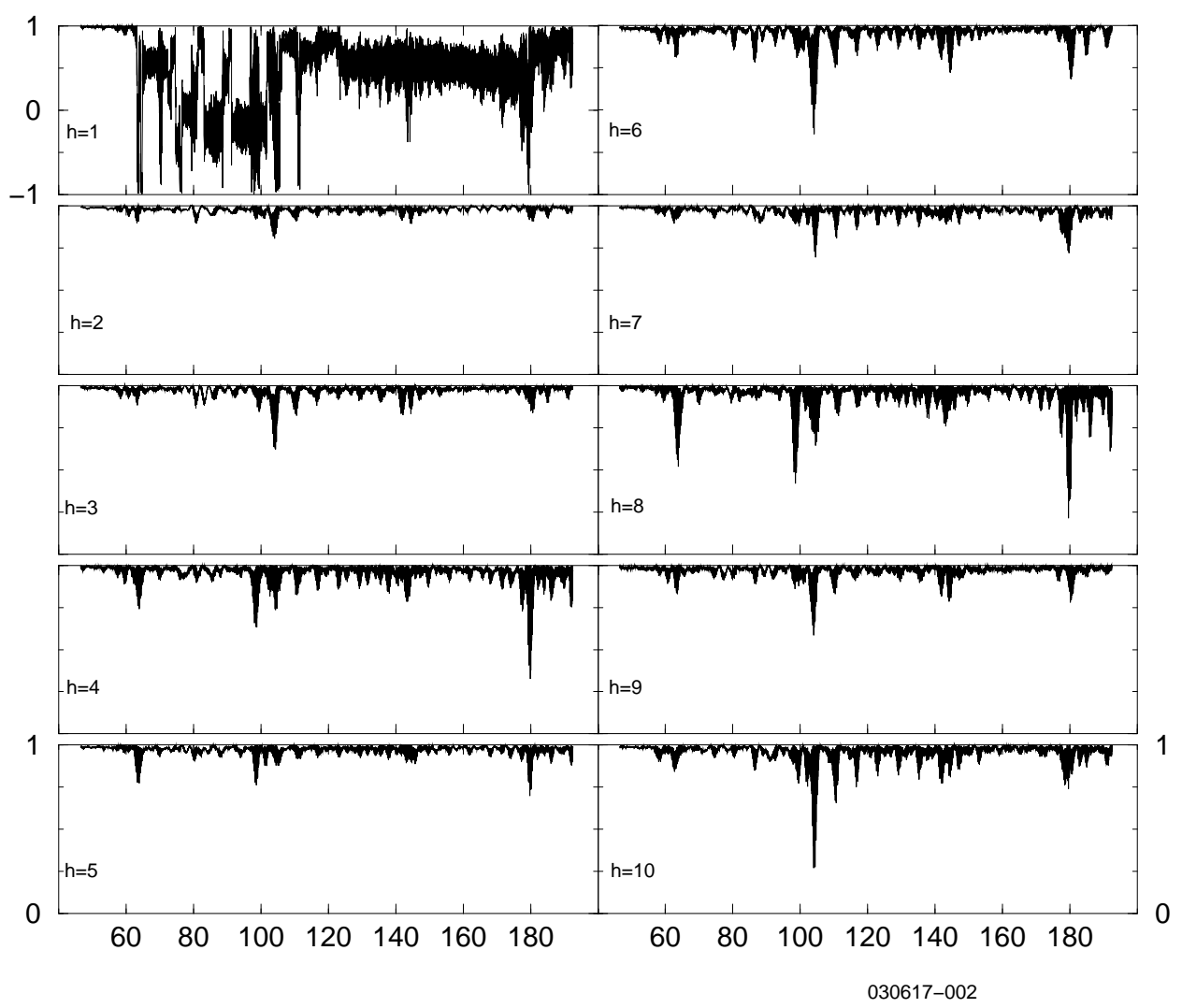

Figure 12: Same as in Fig. 10. $2 \mathrm{~mm}$ COD. One full snake and one partial snake. Same vertical scale in all plots, but the first for $h=1$. 